\title{
Gradient bounds for solutions of nonlinear strictly elliptic equations, applications and extensions
}

\author{
Vinh Duc Nguyen ${ }^{1, *}$ \\ ${ }^{1}$ Faculty of Mathematics and Statistics, Ton Duc Thang University, Ho Chi Minh City, Vietnam
}

\begin{abstract}
In this short manuscript, we briefly recall some well-known methods for obtaining gradient bounds of viscosity solutions for elliptic and parabolic equations. The two methods we focus here are the idea coming from IshiiLions' method applies for strictly elliptic equations and the other one is socalled weak Bernstein's method developed by Barles applies for degenerate equations. We present some results based on these methods and their applications. We also discuss some promising extensions in the future.
\end{abstract}

\section{1 introduction}

In the theory of nonlinear elliptic or parabolic equations, having gradient bounds is very important for proving the existence of solutions. It is even more useful in many applications, for example to singular perturbations problems, long time convergences.

The classical Bernstein's method is the well-known tool to obtain these bounds but the method only applies for smooth solutions.

The key idea of the classical Bernstein's method (cf. Gilbarg and Trudinger[4] and Lions[5]) is the following: if $u: \Omega \subset \mathbb{R}^{N} \rightarrow \mathbb{R}$ is a smooth solution of

$$
-\Delta u=0 \text { in } \Omega
$$

After performing a change of function $v=|D u|^{2}, v$ solves

$$
-\Delta v \leq 0 \text { in } \Omega
$$

The strong maximum principle for elliptic equation says that

$$
\sup _{x \in \Omega} v \leq \sup _{x \in \partial \Omega} v \text { hence } \sup _{x \in \Omega}|D u(x)| \leq \sup _{x \in \partial \Omega}|D u(x)| .
$$

From the above inequality, we have the bound for $|D u(x)|, x \in \Omega$ if we have the bound for $|D u(x)|$ with $x \in \partial \Omega$. With suitable structure conditions on $F$, we can perform the above arguments to obtain gradient bounds for nonlinear elliptic equations:

$$
F\left(x, u, D u, D^{2} u\right)=0, x \in \Omega .
$$

Disadvantage of the classical Bernstein's method: in order to the strong maximum principle holds, $v$ needs to be $C^{2}$ and hence $u$ needs to be $C^{3}$.

\footnotetext{
*e-mail: nguyenducvinh@tdtu.edu.vn
} 
For making the presentation easier, from now we work on quasi-linear elliptic equations. We do not consider boundary conditions to concentrate on structure conditions

$$
-\operatorname{trace}\left(A(x) D^{2} u\right)+H(x, D u)+\epsilon u=0, x \in \mathbb{T}^{N} .
$$

The weak Bernstein's method applies for continuous viscosity solutions developed by Barles [4], is another way to obtain gradient bounds. Instead of the idea of performing a change of function $v=|D u|^{2}$, Barles [4] develops a so called weak Bernstein's method by considering the test function

$$
\Phi(x, y)=u(x)-u(y)-L|x-y|
$$

The goal is to prove that for $L$ large enough, $\max _{x, y \in \bar{\Omega}} \Phi(x, y) \leq 0$ and hence $\|D u\|_{\infty} \leq L$. Surprisingly, the structure conditions of $F$ obtained in Barles [4] are almost the same with the ones in the classical Bernstein's method. used.

Disadvantage of weak Bernstein's method: the strength of ellipticity is not totally

The Ishii-Lions' method introduced in [15], see also [5, 12] allows us to take profit from the strict ellipticity of the equation to control the first order terms. In Ishii-Lions [15] and Barles [3], weak regularity assumptions are assumed over $H$, merely a kind of balance between some Hölder continuity in $x$ and the growth size of $H$ with respect to the gradient, more precisely,

$$
|H(x, p)-H(y, p)| \leq C|x-y \| p|^{3}+C\left(1+|p|^{2}\right) \quad \text { in [3, Assumption (3.4)], }
$$

where $x, y \in \mathbb{T}^{N}, p \in \mathbb{R}^{N}, \tau \in[0,1], C>0$.

These assumptions are designed for subquadratic Hamiltonians(whose growth is less than $|p|^{2}$ ). This is not surprising since it is known that, in general, the ellipticity is not powerful enough to control the first order terms whose growth is more than quadratic [10]. Under these assumptions, the authors obtained gradient bounds which depend on the $L^{\infty}$ norm of the solution.

\section{Disadvantage of Ishii-Lions's method: the strength of first order terms is not totally used.}

Let us mention a recent work of Barles [7] devoted to the study of weak Bernstein's method to local gradient bounds with more general structure conditions for both elliptic and parabolic equations.

\section{Some regularity results}

In [16], we combine the two above methods in some sense. We use the strength from the ellipticity as well as from the strong coercivity of $H$ to allow arbitrary growth of $H$ with respect to the gradient.

In Theorem 2.1, the coercivity assumption (2.1) is the one needed to obtain the Hölder regularity with exponent $\frac{k-2}{k-1}$ in [10]. The main idea is the following:

- showing that the solution is $\gamma$-Hölder continuous for any $\gamma \in\left(\frac{k-2}{k-1}, 1\right)$.

- improving the regularity up to Lipschitz continuity.

Starting with a Hölder exponent equal to $\frac{k-2}{k-1}$ seems crucial to be able to improve the regularity for the class of $H$ we consider which has a strong growth with respect to the gradient. 
Theorem 2.1. Assume $A \geq v I d, v>0$ and $H$ satisfies

$$
\text { there exist constants } k>2, C>0 \text { such that } H(x, p) \geq \frac{1}{C}|p|^{k}-C
$$

and

$$
\left\{\begin{array}{l}
\text { there exist a modulus of continuity } \omega \text { and constants } \alpha \in[0,1], \beta<k-1 \\
\text { such that for all } x, y \in \mathbb{T}^{N}, p \in \mathbb{R}^{N} \\
|H(x, p)-H(y, p)| \leq \omega\left(\left(1+|p|^{\beta}\right)|x-y|\right)|x-y|^{\alpha}|p|^{(k-1) \alpha+k}+o\left(|p|^{k}\right)
\end{array}\right.
$$

where $o\left(|p|^{k}\right) /|p|^{k} \rightarrow 0$ as $|p| \rightarrow+\infty$, uniformly with respect to $x \in \mathbb{T}^{N}$. Then, there exists $K>0$ such that for all $\epsilon>0$, any continuous solution $v^{\epsilon}$ of (1.2) satisfies

$$
\left\|D v^{\epsilon}\right\|_{\infty} \leq K
$$

Assume there exists $k>2$ such that

$$
H(x, p)=a(x)|p|^{k}+K(x, p),
$$

With $a \in C\left(\mathbb{T}^{N}\right)$ and $K(x, p)=o\left(|p|^{k}\right)$, this Hamiltonian satisfies the assumptions of Theorem 2.1 .

Gradient bounds are very useful in singular perturbations problems, long time convergences. Let us state a well-known fact when (2.3) holds as a consequence of strong maximum principle for viscosity solutions proved in [13].

Theorem 2.2. Assume $A \geq v I d, v>0$ and (2.3) holds. Then any bounded continuous solution of

$$
\begin{cases}\frac{\partial u}{\partial t}-\operatorname{trace}\left(A(x) D^{2} u\right)+H(x, D u)=0, & (x, t) \in \mathbb{T}^{N} \times(0,+\infty), \\ u(x, 0)=u_{0}(x), & x \in \mathbb{T}^{N}\end{cases}
$$

converges uniformly to a continuous function $v$ as $t \rightarrow \infty$. Moreover, $v$ is a solution of the equation

$$
-\operatorname{trace}\left(A(x) D^{2} v\right)+H(x, D v)=0
$$

\section{Future extensions}

We wonder if (2.3) and Theorem 2.2 still hold for the following $m$-laplace equation

$$
\epsilon v^{\epsilon}-\operatorname{div}\left(\left|D v^{\epsilon}\right|^{m-2} D v^{\epsilon}\right)+H\left(x, D v^{\epsilon}\right)=0, \quad x \in \mathbb{T}^{N} .
$$

with similar structure conditions. While (2.3) is quite clear to proceed, Theorem 2.2 is quite unclear since we know that the $m$-laplacian term $\operatorname{div}\left(\left|D v^{\epsilon}\right|^{m-2} D v^{\epsilon}\right)$ is degenerate as $D v^{\epsilon}=0$. Let us state the main idea of the proof for (2.3) taken from our work in preparation [21].

- From the super-quadratic structures on first order terms, in [10], the authors proved that

$$
v^{\epsilon} \text { is } \frac{k-m}{k-m+1} \text {. }
$$


- Although the $m$-laplacian term $\operatorname{div}\left(\left|D v^{\epsilon}\right|^{m-2} D v^{\epsilon}\right)$ is degenerate as $D v^{\epsilon}=0$ but when $D v^{\epsilon}$ big, it is strictly elliptic.

- The proof of gradient bounds consists of improving the Holder exponent.

Obtain local gradient bounds for elliptic equations associated with a nonlinear Neumann boundary condition

$$
\begin{cases}-\operatorname{trace}\left(A(x) D^{2} u\right)+H(x, D u)=0, & x \in \Omega, \\ G(x, u, D u)=0, & x \in \partial \Omega .\end{cases}
$$

with similar structure conditions on $H$. Local gradient bounds for superquadratic Hamiltonians are the best we can expect since it is known that the gradients of solutions can blow up at boundary, see [9] and references therein for more results about this phenomenon.

\section{References}

[1] Olivier Alvarez and Martino Bardi. Ergodicity, stabilization, and singular perturbations for Bellman-Isaacs equations. Mem. Amer. Math. Soc., 204(960):vi+77, 2010.

[2] M. Arisawa and P.-L. Lions. On ergodic stochastic control. Comm. Partial Differential Equations, 23(11-12):2187-2217, 1998.

[3] G. Barles. Interior gradient bounds for the mean curvature equation by viscosity solutions methods. Differential Integral Equations, 4(2):263-275, 1991.

[4] G. Barles. A weak Bernstein method for fully nonlinear elliptic equations. Differential Integral Equations, 4(2):241-262, 1991.

[5] G. Barles. $C^{0, \alpha}$-regularity and estimates for solutions of elliptic and parabolic equations by the Ishii \& Lions method. In International Conference for the 25th Anniversary of Viscosity Solutions, volume 30 of Gakuto International Series, Mathematical Sciences and Applications, pages 33-47. Gakkotosho, Tokyo, Japan, 2008.

[6] G. Barles. A short proof of the $C^{0, \alpha}$-regularity of viscosity subsolutions for superquadratic viscous Hamilton-Jacobi equations and applications. Nonlinear Anal., 73(1):31-47, 2010.

[7] G. Barles. Local Gradient Estimates for Second-Order Nonlinear Elliptic and Parabolic Equations by the Weak Bernstein's Method. Submitted.

[8] G. Barles and P. E. Souganidis. Space-time periodic solutions and long-time behavior of solutions to quasi-linear parabolic equations. SIAM J. Math. Anal., 32(6):1311-1323 (electronic), 2001.

[9] Guy Barles and Francesca Da Lio. On the generalized Dirichlet problem for viscous Hamilton-Jacobi equations. J. Math. Pures Appl. (9), 83(1):53-75, 2004.

[10] I. Capuzzo Dolcetta, F. Leoni, and A. Porretta. Hölder estimates for degenerate elliptic equations with coercive Hamiltonians. Trans. Amer. Math. Soc., 362(9):4511-4536, 2010.

[11] P. Cardaliaguet and L. Silvestre. Hölder continuity to Hamilton-Jacobi equations with superquadratic growth in the gradient and unbounded right-hand side. Comm. Partial Differential Equations, 37(9):1668-1688, 2012.

[12] M. G. Crandall, H. Ishii, and P.-L. Lions. User's guide to viscosity solutions of second order partial differential equations. Bull. Amer. Math. Soc. (N.S.), 27(1):1-67, 1992.

[13] F. Da Lio. Remarks on the strong maximum principle for viscosity solutions to fully nonlinear parabolic equations. Commun. Pure Appl. Anal., 3(3):395-415, 2004. 
[14] David Gilbarg and Neil S. Trudinger. Elliptic partial differential equations of second order. Classics in Mathematics. Springer-Verlag, Berlin, 2001. Reprint of the 1998 edition.

[15] H. Ishii and P.-L. Lions. Viscosity solutions of fully nonlinear second-order elliptic partial differential equations. J. Differential Equations, 83(1):26-78, 1990.

[16] O. Ley and V. D. Nguyen. Lipschitz regularity results for nonlinear strictly elliptic equations and applications. Journal of Differential Equations 263, 4324-4354, 2017.

[17] O. Ley and V. D. Nguyen. Gradient bounds for nonlinear degenerate parabolic equations and application to large time behavior of systems. Nonlinear Anal., 130:76-101, 2016.

[18] N. V. Krylov. Lectures on elliptic and parabolic equations in Hölder spaces, volume 12 of Graduate Studies in Mathematics. American Mathematical Society, Providence, RI, 1996.

[19] P.-L. Lions. Generalized solutions of Hamilton-Jacobi equations. Pitman (Advanced Publishing Program), Boston, Mass., 1982.

[20] P.-L. Lions, B. Papanicolaou, and S. R. S. Varadhan. Homogenization of HamiltonJacobi equations. Unpublished, 1986.

[21] V. D. Nguyen. Regularity results and long time behavior for solutions of $p$-laplace equations. In preparation. 